In Crescendo, 2020; 11(1): 51-66

Fecha de recepción: 07/01/2020

Fecha de aceptación: 26/03/2020

\title{
CALIDAD DEL GASTO Y MANEJO PRESUPUESTAL EN LA UNIVERSIDAD NACIONAL DE UCAYALI, CALLERÍA, 2019
}

\author{
QUALITY OF SPENDING AND BUDGET MANAGEMENT AT THE \\ NATIONAL UNIVERSITY OF UCAYALI, CALLERÍA, 2019
}

María M. Castillo ${ }^{1}$

\section{RESUMEN}

Esta tesis titulada Calidad del gasto y manejo presupuestal en la Universidad Nacional de Ucayali, Callería, 2019, cuyas variables se sustentaron en la teoría del efecto burocracia y la del presupuesto principalmente, tuvo como objetivo determinar si existe relación significativa entre calidad de gasto y manejo presupuestal según operadores financistas de la Universidad Nacional de Ucayali, 2019. Investigación descriptiva, correlacional y transeccional, cuya población muestral estuvo conformada por 30 operadores financistas de la Universidad Nacional de Ucayali, a quienes se les aplicaron cuestionarios para cada variable para obtener datos acerca de su percepción. Entre los resultados se obtuvo que se percibe la calidad de gasto en un nivel medio, a diferencia del manejo presupuestal que se percibió muy deficiente, esto ocasionado por la mala distribución y asignación del gasto, que se traduce en el deficiente manejo de insumos y producto que ocasiona ausencia de calidad y resultados ideales. La principal conclusión a la que se arribó fue que no existe relación significativa entre calidad de gasto y manejo presupuestal según operadores financistas de la Universidad Nacional de Ucayali, 2019, ya que, el resultado Rho $=0.184$ indica una correlación positiva débil y el $\mathrm{p}$-valor $=0.331$ indica que la correlación no es significativa.

PALABRAS CLAVE: Gasto, presupuesto, gestión, calidad.

1 Contador Público Colegiado Certificado, Auditor Independiente, Magíster en Administración de la Educación, Ex Docente Tutor Investigador de la Escuela Profesional de Contabilidad de la ULADECH Católica- sede Pucallpa. Docente de la Universidad Nacional de Ucayali. Egresado de la Escuela de Posgrado de la Universidad César Vallejo-Doctorado en Gestión Pública y Gobernabilidad. 


\begin{abstract}
This thesis entitled Quality of expenditure and budgetary management at the Universidad Nacional de Ucayali, Callería, 2019, whose variables were based on the theory of bureaucracy and budget theory, mainly aimed to determine if there is a significant relationship between quality of expenditure and budgetary management budgetary management according to financial administrators of the Universidad Nacional de Ucayali, 2019. Descriptive, correlational and transectional research, whose sample population was made up of 30 financial administrators of the Universidad Nacional de Ucayali, to whom questionnaires were applied for each variable for get data about your perception. Among the results it was obtained that the quality of expenditure is perceived at a medium level, unlike the budget management that was perceived as very poor, this caused by the poor distribution and allocation of expenditure, which translates into the poor management of inputs and products that causes absence of quality and ideal results. The main conclusion reached was that there is no significant relationship between quality of expenditure and budget management according to financial administrators of the Universidad Nacional de Ucayali, 2019, since the result $\mathrm{Rho}=0.184$ indicates a weak positive correlation and the $\mathrm{p}$-value $=0.331$ indicates that the correlation is not significant.
\end{abstract}

KEY WORDS: Expenditure, budget, management, quality.

\title{
INTRODUCCIÓN
}

En cuanto a la realidad problemática, el estudio de las formas en que una entidad pública realiza el gasto de lo que el Estado le designa para la gestión y el cómo maneja su presupuesto es de suma importancia para quienes laboran en ella, puesto que la fluidez del trabajo que realizan depende de en qué medida pueden ser atendidos a nivel salarial y de suministro de todo aquello que signifique un soporte para el trabajo, más aún si se brinda un servicio en el que el trabajador es el primero que se contacta con el usuario y a quien van dirigido los reclamos cuando no son satisfechas las demandas o cuando no existe la logística necesaria para atenderlo.

En este sentido, se requiere conocer si quienes se encargan del ordenamiento y efectividad del gasto público y los que diseñan o formulan el presupuesto de una institución están realizando bien su labor desde la perspectiva de quienes de manera directa son los que reciben el impacto de las acciones de gestión, así, será posible tomar decisiones acerca de la superación de falencias que puedan evidenciarse o de, si se percibe que se está haciendo bien, optimizar el desarrollo de estas acciones administrativas. 
Finalmente, surge el interés por realizar esta investigación, porque es una motivación sustancial la de aportar con un estudio serio y significativo, en el que más allá de los datos numéricos, se recoja información sobre características y cualidades evaluadas por expertos en lo referido a presupuesto, de modo que toda decisión de mejora que una vez socializados los resultados del estudio se adopte, se caracterice por la pertinencia y la oportunidad.

En casi toda las instituciones públicas del país existe una elevada crisis en el manejo del presupuesto que se les asigna a través de la ley anual del presupuesto que se ve reflejada por su ineficiencia e ineficacia en la gestión y logro de sus objetivos, al extremo de generar esta mala gestión presupuestal insatisfacción dentro de los propios trabajadores de las entidades, quienes no ven que la institución sea capaz de satisfacer sus necesidades básicas que les permita cumplir con sus labores; generando este malestar baja productividad y la no identificación de los trabajadores con la institución.

La calidad del gasto público abarca todos los elementos que garantizan el uso eficaz y eficiente de los recursos públicos, que tiene como objetivo elevar el potencial de crecimiento de la economía, asegurando grados crecientes de equidad distributiva. La medición de la calidad del gasto público debe incorporar múltiples factores que inciden en el logro de los objetivos planteados por el estado.

En la región Ucayali se cuestiona el manejo presupuestal, más aún cuando existen obras públicas que, pese a ser iniciadas en gestiones previas, no han sido concluidas por quienes dirigen el gobierno de la Región, son muy pocas las obras realizadas hasta la actualidad lo que da cuenta que no responden a las reales necesidades de los pobladores, pudiéndose inferir que la calidad del gasto es deficiente.

Es así que en la Universidad Nacional de Ucayali se advierte varias falencias en la ejecución del presupuesto asignado ya sea porque la institución no está priorizando las necesidades más urgentes, por la falta de capacitación del personal encargado del manejo del presupuesto asignado o por el mal manejo de dicho presupuesto, por ejemplo, cuando se asignan dinero de la institución para cubrir dos veces la misma necesidad.

Este mal manejo presupuestal percibido genera insatisfacción laboral en el personal administrativo de la Universidad Nacional de Ucayali, quienes se ven directamente afectados por el ineficiente manejo presupuestal porque sus necesi- 
dades básicas para que puedan realizar sus labores no pueden ser cubiertas a cabalidad y es notorio el malestar, tanto del trabajador como del público usuario que, mayoritariamente son los alumnos.

En Chile, Letelier (2016) concluye que "La estrategia de estimación utilizada fue Generalized Method of Moments (GMM), debido a que explota la variación de series de tiempo de los datos, controla por efectos inobservables específicos al país, permite la inclusión de rezagos de la variable dependiente como regresores y controla por la endogeneidad de las variables explicativas. Obteniendo así estimadores consistentes y eficientes." (pág. 45).

González \& Rodríguez (2013) concluyó que la Universidad de Cartagena con un incremento de sus ingresos del 2,8\% y una disminución 5.65\% en sus gastos en el año 2010 respecto al 2009, obtuvo un avance en cuanto a cobertura, logrando un incremento en el número de estudiantes matriculados en pregrado de $27,7 \%$ pese al aumento en la deserción estudiantil del 1,1\% que no representa mayor significación, mientras que la universidad con un aumento en sus ingresos del 1,57\% y con un aumento considerado de los gastos de 21,39\% en el 2011 respecto al año anterior, obtuvo un aumento de los matriculados de pregrado en un $21,51 \%$, pero con un aumento en la deserción del 4,9\%. Lo que muestra la ineficiencia con que se manejaron los recursos en el 2011 respecto al 2010 en materia de cobertura.

Cañas (2015) concluyó "La estrategia de estimación utilizada fue Generalized Method of Moments (GMM), debido a que explota la variación de series de tiempo de los datos, controla por efectos inobservables específicos al país, permite la inclusión de rezagos de la variable dependiente como regresores y controla por la endogeneidad de las variables explicativas. Obteniendo así estimadores consistentes y eficientes." (Pág. 299)

Además, en el ámbito nacional, en nuestro país se realizaron múltiples investigaciones sobre el tema mencionado, después de una investigación minuciosa se eligió los siguientes antecedentes, cuyas conclusiones dan veracidad a la presente investigación.

Tal es el caso de Rodríguez (2017), entre sus resultados expone que la asignación y ejecución del presupuesto repercute en la operatividad de la Policía Nacional del Perú en razón que este es producto de la programación y asignación de recursos anuales, sin embargo, la ejecución no necesariamente representa la verdadera necesidad de la organización policial porque en promedio anualmente 
existe un déficit presupuestal de apertura de poco más de 2,000 millones de soles, es frecuente que la programación formulada sea modificada por diferentes motivos, pueden ser motivos coyunturales o políticos, puede haber nuevas prioridades o puede ser que se presenten imprevistos que deben ser atendidos, lo que determina la transferencia de recursos de una partida a otra, signo ineludible de debilidad en la programación y la asignación, concluyó que la asignación presupuestal, su interrelación con los bienes públicos y con la eficiencia del gasto no se articulan adecuadamente.

Asimismo, se puede colegir la existencia de una incongruencia de estos factores, por cuanto, en la medida que el presupuesto asignado a la Policía Nacional del Perú se ha incrementado, este no necesariamente se ha visto reflejado en la eficiencia de la distribución presupuestal y el gasto generado, y fundamentalmente en el resultado, o dicho de otra manera, el presupuesto no se conoce en su verdadera dimensión por no haber sido medido o por no evaluarse el impacto de la ejecución del gasto público, no se conoce en qué medida la operatividad de las unidades policiales ha mejorado en su capacidad de respuesta al llamado de la ciudadanía y a resolver el problema público de inseguridad.

Ponce (2007) entre sus resultados expuso que progresivamente se nota eficacia en el gasto público en la mayoría de departamentos del Perú, sobre todo en Tacna, pues el rendimiento en Comunicación y Matemática se ha elevado, en varios departamentos entre 2 y 3 puntos porcentuales, pero en algunos se ha elevado el rendimiento en estas materias de manera sustancial. Es precisamente Tacna la que ocupa, en cuanto a mejoras, el primer puesto en el país concluyó que existe una relación positiva entre los logros alcanzados en el ámbito en términos del rendimiento académico y el nivel de financiamiento otorgado por el Estado para la educación pública, tanto para el nivel del sexto de primaria como del quinto de secundaria.

Arbildo (2018) concluyó que "La administración financiera gubernamental y la utilización de los fondos públicos son percibidos en un nivel regular por los trabajadores administrativos del Ministerio Público-Fiscalía de la Nación, Distrito Fiscal de Ucayali, 2018" (p. 42), lo que da cuenta de la necesidad de reajustar o repensar los procedimientos que se siguen en el manejo de los fondos que derivarán en el gasto público de esta entidad.

Además, Castro, Villacorta \& Caja (2017) concluyó que "La relación es positiva muy baja y no significativa entre la remuneración y la calidad del servicio 
en el Hospital Regional de Pucallpa, 2016, en razón de haber obtenido el valor de relación de $(0.042)$, y de significancia de 0.702 , por lo que se determina que la remuneración de los trabajadores del Hospital Regional de Pucallpa, no influye positivamente en la calidad del servicio que brinda, toda vez que en la actualidad los trabajadores administrativos y asistenciales se encuentran en constantes paralizaciones de sus actividades por sus bajas remuneraciones, lo que trae como consecuencia un mal servicio al usuario.” (pág. 62)

Criollo (2018) concluyó que "entre la calidad del servicio asistencial y la gestión comunitaria en la percepción de los usuarios del Seguro Integral de Salud, Hospital Amazónico-Yarinacocha, Ucayali, 2018, existe una relación positiva media de acuerdo al resultado de la prueba de Tau b de Kendall .719." (p. 66), lo que refiere que el usuario recibe el impacto de la gestión presupuestal, pues, si recibe atención en la comunidad a través de los programas de impacto social, entonces el manejo presupuestal y la calidad del gasto es saludable.

Asimismo, Panduro (2018) concluyó que "La administración está comprometida con la mejora continua, sin embargo, en la práctica no usan herramientas de calidad de gestión como procesos normalizados o técnicas para solución de problemas. El 50,0\% no ha definido la misión, visión y valores." (pág. 56)

Patiño (2018) concluyó que "Entre la gestión presupuestal y satisfacción laboral en la percepción del personal administrativo, Ministerio Público sede Ucayali, 2018, existe una relación positiva muy fuerte según el resultado de Rho de Spearman (,880)." (p. 66), es decir que, a mejor gestión presupuestal, mayor satisfacción laboral.

En cuanto la variable calidad de gasto, para el Sistema Nacional de Presupuesto Ley № 28411, los gastos públicos son el conjunto de erogaciones que, por concepto de gasto corriente, gasto de capital y servicio de deuda, realizan las Entidades con cargo a los créditos presupuestarios aprobados en los presupuestos respectivos, para ser orientados a la atención de la prestación de los servicios públicos y acciones desarrolladas por las Entidades de conformidad con sus funciones y objetivos institucionales.

Entre las teorías que se relacionan esencialmente con la calidad del gasto, explica Bulacio (2000), la Ley de Wagner. Este teórico precisó que "a medida que aumenta el ingreso per cápita en los países en vías de industrialización, el Estado aumenta su participación en el producto bruto. Dio tres razones para explicar este fenómeno. En primer lugar, se produciría un aumento de las funciones adminis- 
trativas y protectoras del Estado con la consecuente sustitución de la actividad privada por la pública. Asimismo, sostuvo que el aumento de la densidad de población y la urbanización son causales del aumento del Gasto debido a la necesidad de regulación económica. En segundo lugar, Wagner manifestó que aumentarían los gastos culturales y de bienestar, especialmente los relativos a educación y redistribución de la renta. Las razones que dio en este aspecto no fueron muy claras pero el resultado podría interpretarse en el sentido que estos bienes son superiores y por lo tanto su elasticidad con respecto al ingreso mayor a la unidad. Por último, Wagner sugirió que el aumento de la industrialización daría lugar a la aparición de grandes monopolios que requerirían la presencia del Estado para su regulación económica o bien para hacerse cargo de ellos." (pág. 2)

Acerca de la teoría del efecto burocracia, según esta teoría, la explicación para el aumento del gasto puede encontrarse analizando la conducta de los funcionarios públicos. Esta teoría, a la que podemos denominar efecto burocracia, argumenta que los funcionarios actúan en su propio interés maximizando la cantidad de recursos que ellos pueden manejar. Cuentan a su favor que disponen de información privilegiada, circunstancia que les posibilita lograr su propósito. Esta conducta maximizadora de los funcionarios se refleja en el agregado en una tendencia expansiva del gasto público.

Son dimensiones de la variable calidad de gasto, considerando lo señalado por Salvador y Deming (citados por Carranza, 2017) y el Decreto Legislativo $\mathrm{N}^{\circ}$ 1440 -Decreto Legislativo del Sistema Nacional de Presupuesto Público-, la eficacia, la eficiencia, la legalidad y la veracidad.

Respecto de la variable manejo presupuestal, esta se define, según la Dirección general de presupuesto público (2011), como la práctica de dirigir una entidad para definir el volumen y en valor las previsiones de actividad de la organización de manera anual y posteriormente realizarlas previniendo y apoyándose en la estructura de la organización teniendo en cuenta costos, ingresos, egresos, los beneficios y la inversión. Se debe establecer en un centro laboral y programas de prevención para las actividades de gestión presupuestaria relacionada con la planificación, la organización de la contabilidad y la estructura de la organización.

La gestión presupuestal se mide en cantidades de números, que se toman de las contabilidades que se lleven de manera analítica, referidas a las actividades que realizará de modo que se preparen los datos y poder llevar un control. 
La teoría del presupuesto, acerca de la cual Albi et al. (1992) indica que el presupuesto público es un reflejo de gastos e ingresos que se prevén en un determinado ámbito de las instituciones públicas en tiempos establecidos. Es decir, que permite medir en intervalos anuales los presupuestos que se van a destinar a distintas partidas de gasto, además de prever la recaudación y fondos que provienen de diversas fuentes de ingreso. El presupuesto público es el documento en el que se declaran las intenciones del estado para el ejercicio anual, sintetizando desde una perspectiva de economía el plan de actuación que va a dirigir la gestión, para esto se deben responder a preguntas como ¿Qué hacer? Las políticas que se van a realizar en el año; ¿Quién puede gastar y hasta dónde? Se realiza la clasificación de los gastos; ¿Para qué se va a gastar? La clasificación y programación del presupuesto; ¿Cómo se hará el gasto? La clasificación que se hará del gasto público; ¿Cómo se va a recaudar y conseguir ingresos? Se clasifica los ingresos necesarios.

Las características fundamentales son:

- El carácter jurídico del documento presupuestario: el presupuesto es la previsión que se realiza por norma y debe ser aprobado en el congreso y obligatorio en el estado, siendo relevante para prever los gastos, puesto que se puede establecer el máximo límite de los que se pueden realizar.

- El presupuesto es un documento de elaboración y ejecución periódica; expresado en contabilidad, lo que significa que se adoptan diversas estructuras para clasificar los ingresos y gastos de manera equilibrada. El equilibrio de la contabilidad radica en la partida doble en la que cada operación contable tiene partidas y contrapartidas.

- El presupuesto constituye un acto de previsión, permite estimar lo que deben ser los ingresos y gastos, de esto parte lo importante que son las técnicas e hipótesis de previsión en las que se desarrolla.

- Distinción entre información presupuestaria y contable; los sistemas de contabilidad tienen un objetivo que es el establecimiento de una imagen real de la situación económica de la institución, para lo que se necesita una evolución de la situación económica. Mientras que los presupuestos públicos se limitan a los ingresos y gastos que repercuten en el tesoro público, estos no recogen los gastos posibles que puedan producirse en el ejercicio económico y que por el contrario sí se refleja en la contabilidad general. 
En cuanto a los principios presupuestarios, estos son:

- Principios políticos; son los criterios normativos que son un instrumento de equilibrio de poderes y de distribución de las competencias del Estado.

- Principio de competencia; es el Congreso el encargado de aprobar y promulgar el presupuesto, al ejecutivo le compete ejecutarlo y al Poder Judicial fiscalizar.

- Principio de universalidad o de integridad; en el presupuesto se debe tener en cuenta los ingresos y gatos de las actividades financieras bien explicados.

- Principio de unidad presupuestaria; el presupuesto es único y por ende debe estar excluida de la propagación de presupuestos especiales. (Albi, et al. 1992)

Respecto al marco legal del tema que se aborda, la Constitución Política del Perú y, específicamente, la Ley № 28411 del Sistema Nacional de Presupuesto, señalan la responsabilidad y cualidad del presupuesto y la calidad del gasto, por lo que realizar el presente estudio enmarcándose en lo prescrito por esta normativa es una forma de aportar al tema de investigación vinculándose a lo normativo, más aun siendo presupuesto y gasto en la Universidad Nacional de Ucayali; en este sentido, la Constitución peruana refiere, en el artículo $16^{\circ}$ : "Se da prioridad a la educación en la asignación de recursos ordinarios del Presupuesto de la República." (p. 8). El artículo $77^{\circ}$ manifiesta:

"La administración económica y financiera del Estado se rige por el presupuesto que anualmente aprueba el Congreso. La estructura del presupuesto del sector público contiene dos secciones: gobierno central e instancias descentralizadas. [...] El presupuesto asigna equitativamente los recursos públicos, su programación y ejecución responden a los criterios de eficiencia de necesidades sociales básicas y de descentralización. Corresponden a las respectivas circunscripciones, conforme a ley, recibir una participación adecuada del total de los ingresos y rentas obtenidos por el Estado en la explotación de los recursos naturales en cada zona en calidad de canon." (pp. 20,21)

La Ley General del Sistema Nacional de Presupuesto $N^{\circ} 28411$, en su artículo 12, manifiesta que, en cuanto a los gastos públicos, estos: 
"se estructuran siguiendo las Clasificaciones Institucional, Económica, Funcional Programática y Geográfica” (p. 7); además, en el artículo 22 refiere: "Las Leyes de Presupuesto del Sector Público, aprobadas por el Congreso de la República, así como los demás presupuestos, constituyen el total del crédito presupuestario, que comprende el límite máximo de gasto a ejecutarse en el año fiscal. A la Ley de Presupuesto del Sector Público se acompañan los estados de gastos del presupuesto que contienen los créditos presupuestarios estructurados siguiendo las clasificaciones: Institucional, Funcional Programático, Grupo Genérico de Gasto y por Fuentes de Financiamiento." (p. 12)

Las dimensiones de la variable manejo presupuestal, según las definiciones del Ministerio de Economía y Finanzas, son insumos, producto, calidad y resultado e impacto.

El objetivo del estudio es determinar si existe relación significativa entre calidad de gasto y manejo presupuestal según los operadores financistas de la Universidad Nacional de Ucayali, 2019.

\section{MÉTODOS}

La población muestral estuvo constituida por 30 operadores administrativos financistas de la Universidad Nacional de Ucayali, Ucayali: 8: Administradores de Facultades. 2: Operadores de la Escuela de Posgrado. 4: Operadores de la Dirección General de Administración. 2: Operadores de la Oficina de Contabilidad. 6: Operadores de la Oficina de Abastecimiento. 6: Operadores de la Oficina de Remuneraciones. 12: Operadores de Caja. La técnica utilizada fue la encuesta, que permitió reunir, de manera sistemática, datos sobre el tema de estudio, a través del contacto directo e indirecto con los trabajadores y usuarios que formarán parte de la muestra. Los instrumentos de recolección de datos, validez y confiabilidad, utilizados fueron dos cuestionarios. Se procedió a la validación de los instrumentos mediante el juicio de expertos, así como la aplicación del coeficiente de fiabilidad Alfa de Cronbach, para determinar la aplicabilidad de los instrumentos.

\section{Métodos de análisis de datos}

El proceso de análisis de datos fue posible mediante la aplicación de métodos estadísticos, tal como la estadística descriptiva. Se elaboraron tablas de frecuencias y porcentajes con sus respectivos gráficos. Estas tablas serán unidimen- 
sionales y bidimensionales. Se realizó una prueba de hipótesis que, según el resultado de la prueba de normalidad Kolmogorov-Smirnov, fue Rho Spearman.

\section{RESULTADOS}

Tabla 1

DISTRIBUCIÓN DE FRECUENCIAS: CALIDAD DE GASTO Y DIMENSIONES

\begin{tabular}{|c|c|c|c|c|c|c|c|c|c|c|}
\hline & \multicolumn{2}{|c|}{ Calidad de gasto } & \multicolumn{2}{|c|}{ Eficacia } & \multicolumn{2}{|c|}{ Eficiencia } & \multicolumn{2}{|c|}{ Legalidad } & \multicolumn{2}{|c|}{ Veracidad } \\
\hline & Recuento & $\%$ & Recuento & $\%$ & Recuento & $\%$ & Recuento & $\%$ & Recuento & $\%$ \\
\hline Bajo & 2 & 6.7 & 3 & 10.0 & 3 & 10.0 & 6 & 20.0 & 4 & 13.3 \\
\hline Medio & 21 & 70.0 & 18 & 60.0 & 17 & 56.7 & 21 & 70.0 & 21 & 70.0 \\
\hline Alto & 7 & 23.3 & 9 & 30.0 & 10 & 33.3 & 3 & 10.0 & 5 & 16.7 \\
\hline Total & 30 & 100.0 & 30 & 100.0 & 30 & 100.0 & 30 & 100.0 & 30 & 100.0 \\
\hline
\end{tabular}

Fuente: Base de datos.

La tabla muestra que, del total de la muestra, en cuanto a la variable Calidad de gasto, $63.3 \%$ la percibe en el nivel Medio, 30\% en el nivel Alto y $6.7 \%$ en el nivel Bajo; respecto a la dimensión Eficacia, $60 \%$ la percibe en el nivel Medio, $30 \%$ en el nivel Alto y $10 \%$ en el nivel Bajo; en cuanto a la dimensión Eficiencia, $56.7 \%$ la percibe en el nivel Medio, 33.3\% en el nivel Alto y $10 \%$ en el nivel Bajo; respecto a la dimensión Legalidad, 70\% la percibe en el nivel Medio, 20\% en el nivel Bajo y 10\% en el nivel Alto; y en cuanto a la dimensión Veracidad, 70\% la percibe en el nivel Medio, $16.7 \%$ en el nivel Alto y $13.3 \%$ en el nivel Bajo, en la sede de estudio.

Tabla 2

DISTRIBUCIÓN DE FRECUENCIAS: MANEJO PRESUPUESTAL Y DIMENSIONES

\begin{tabular}{|c|c|c|c|c|c|c|c|c|c|c|}
\hline & \multicolumn{2}{|c|}{$\begin{array}{c}\text { Manejo } \\
\text { presupuestal }\end{array}$} & \multicolumn{2}{|c|}{ Insumo } & \multicolumn{2}{|c|}{ Producto } & \multicolumn{2}{|c|}{ Calidad } & \multicolumn{2}{|c|}{$\begin{array}{l}\text { Resultado e } \\
\text { impacto }\end{array}$} \\
\hline & $\mathrm{fi}$ & $\%$ & $\mathrm{fi}$ & $\%$ & $\mathrm{fi}$ & $\%$ & $\mathrm{fi}$ & $\%$ & $\mathrm{fi}$ & $\%$ \\
\hline Muy deficiente & 12 & 40,0 & 11 & 36,7 & 12 & 40,0 & 11 & 36,7 & 11 & 36,7 \\
\hline Deficiente & 9 & 30,0 & 12 & 40,0 & 12 & 40,0 & 12 & 40,0 & 12 & 40,0 \\
\hline Regular & 9 & 30,0 & 7 & 23,3 & 3 & 10,0 & 5 & 16,7 & 4 & 13,3 \\
\hline Eficiente & 0 & 0,0 & 0 & 0,0 & 3 & 10,0 & 2 & 6,7 & 2 & 6,7 \\
\hline Muy eficiente & 0 & 0,0 & 0 & 0,0 & 0 & 0,0 & 0 & 0,0 & 1 & 3,3 \\
\hline Total & 30 & 100,0 & 30 & 100,0 & 30 & 100,0 & 30 & 100,0 & 30 & 100,0 \\
\hline
\end{tabular}

Fuente: Base de datos. 
La tabla muestra que, del total de la muestra, en cuanto a la variable Manejo presupuestal, $40 \%$ la percibe en el nivel Muy deficiente, 30\% en el nivel Deficiente y $30 \%$ en el nivel Regular; respecto a la dimensión Insumo, 40\% la percibe en el nivel Deficiente, $36.7 \%$ en el nivel Muy deficiente y 23.3\% en el nivel Regular; en cuanto a la dimensión Producto, sendos $40 \%$ la percibe en los niveles Muy deficiente y Deficiente, y sendos 10\% en los niveles Regular y Eficiente; respecto a la dimensión Calidad, $40 \%$ la percibe en el nivel Deficiente, $36.7 \%$ en el nivel Muy deficiente, $16.7 \%$ en el nivel Regular y $6.7 \%$ en el nivel Eficiente; y en cuanto a la dimensión Resultado e impacto, 40\% la percibe en el nivel Deficiente, $36.7 \%$ en el nivel Muy deficiente, 13.3\% en el nivel Regular, 6.7\% en el nivel Eficiente y 3.3\% en el nivel Muy eficiente.

Tabla 3

RELACIÓN: CALIDAD DE GASTO*MANEJO PRESUPUESTAL

\begin{tabular}{|c|c|c|c|c|c|c|}
\hline & & & \multicolumn{3}{|c|}{ Gestión presupuestal } & \multirow{2}{*}{ Total } \\
\hline & & & Muy deficiente & Deficiente & Regular & \\
\hline \multirow[t]{6}{*}{ Calidad de gasto } & Bajo & Recuento & 2 & 0 & 0 & 2 \\
\hline & & $\%$ del total & 6.7 & 0.0 & 0.0 & 6.7 \\
\hline & Medio & Recuento & 8 & 5 & 8 & 21 \\
\hline & & $\%$ del total & 26.7 & 16.7 & 26.7 & 70.0 \\
\hline & Alto & Recuento & 2 & 4 & 1 & 7 \\
\hline & & $\%$ del total & 6.7 & 13.3 & 3.3 & 23.3 \\
\hline \multirow[t]{2}{*}{ Total } & & Recuento & 12 & 9 & 9 & 30 \\
\hline & & $\%$ del total & 40.0 & 30.0 & 30.0 & 100.0 \\
\hline
\end{tabular}

Fuente: Base de datos.

La tabla muestra que, los mayores porcentajes de la muestra, representados por sendos 26.7\%, perciben la variable Calidad de gasto en el nivel Medio, mientras que perciben la variable Manejo presupuestal en los niveles Muy deficiente y Regular, en la sede de estudio. 
Tabla 4

CORRELACIONES: HIPÓTESIS GENERAL

\begin{tabular}{|c|c|c|c|c|}
\hline & & & $\begin{array}{c}\text { Calidad de } \\
\text { gasto }\end{array}$ & $\begin{array}{c}\text { Manejo } \\
\text { presupuestal }\end{array}$ \\
\hline \multirow[t]{6}{*}{ Rho de Spearman } & \multirow[t]{3}{*}{ Calidad de gasto } & Coeficiente de correlación & 1,000 & ,184 \\
\hline & & Sig. (bilateral) & $\cdot$ & ,331 \\
\hline & & $\mathrm{N}$ & 30 & 30 \\
\hline & \multirow[t]{3}{*}{ Manejo presupuestal } & Coeficiente de correlación & , 184 & 1,000 \\
\hline & & Sig. (bilateral) & ,331 & \\
\hline & & $\mathrm{N}$ & 30 & 30 \\
\hline
\end{tabular}

Fuente: Base de datos.

El resultado Rho $=0.184$ indica una correlación positiva débil y el $\mathrm{p}$-valor $=$ 0.331 indica que la correlación no es significativa, por lo tanto, se rechaza la hipótesis alterna y se acepta la hipótesis nula, es decir, no existe relación significativa entre calidad de gasto y manejo presupuestal según administrativos financistas de la Universidad Nacional de Ucayali, 2018.

\section{DISCUSIÓN}

Este capítulo se realiza debido a la necesidad de responder a la pregunta de investigación, que pretende conocer si existe relación significativa entre calidad de gasto y manejo presupuestal según operadores financistas de la Universidad Nacional de Ucayali, 2019, relación que se identificó y cuya significatividad se determinó después del análisis de los resultados de los datos recolectados.

La tabla 1 nos muestra que son percibidas en el nivel Medio, es decir, los administrativos refieren en su mayoría que existe coherencia entre el monto de la ejecución presupuestaria, PIA y PIM, sin embargo, no se percibe ideal la entrega y distribución de recursos y pagos en general. En cuanto a la tabla 2, acerca de la variable manejo presupuestal y sus dimensiones, estas son percibidas en general en el nivel Muy deficiente y deficiente, demostrando que desde la asignación de insumos y producto afecta en la calidad y los resultados.

Este resultado coincide con el obtenido por Rodríguez (2017) que concluyó que la asignación presupuestal, su interrelación con los bienes públicos y con la eficiencia del gasto no se articulan adecuadamente; asimismo, se puede colegir la existencia de una incongruencia de estos factores. 
Además, en Chile, Letelier (2016) concluye que, aplicando ciertos sistemas apropiados y modernos puede mejorarse la eficiencia y la eficacia del gasto público, haciendo más viable y pertinente el trabajo presupuestal

La tabla 3 corrobora la marcada diferencia de niveles de las tablas anteriores, debido a que confirma la intersección de la mayoría entre la variable calidad de gasto, con semejantes resultados en sus dimensiones -eficacia, eficiencia, legalidad y veracidad-percibidas en el nivel Medio, y la percepción de la variable manejo presupuestal en el nivel muy deficiente, en este sentido, se corrobora la deficiencia en la entrega y distribución de recursos estipulada en la calidad de gasto, sin embargo, esta no ocasionaría el nivel muy deficiente percibido del manejo presupuestal.

Es así que Ponce (2007), en un estudio cualitativo sobre el gasto público en educación en Perú, ha observado que cuando se prioriza ciertos aspectos esenciales, en este caso, educación, de manera correcta, puede mejorarse la situación de los indicadores correctos en función de la ejecución presupuestal.

Finalmente, la tabla 4 confirma lo colegido en la tabla 3, mostrando que no existe correlación significativa entre la variable calidad de gasto, sus dimensiones -eficacia y eficiencia-y la variable manejo presupuestal.

Patiño (2018) concluye que una buena gestión presupuestal produce una mayor satisfacción laboral; al generalizar esta realidad se tiene que se satisface las necesidades e intereses de los diferentes segmentos de la nación y de las regiones.

Esto podría originarse por lo referido por Bulacio (2000), quien afirma que los funcionarios actúan en su propio interés maximizando la cantidad de recursos que ellos pueden manejar, dado que disponen de información privilegiada, circunstancia que les posibilita lograr su propósito. Esta conducta maximizadora de los funcionarios se refleja en el agregado en una tendencia expansiva del gasto público.

\section{CONCLUSIONES}

No existe relación significativa entre calidad de gasto y manejo presupuestal según operadores financistas de la Universidad Nacional de Ucayali, 2019.

Tampoco existe relación significativa entre las dimensiones eficacia, eficiencia, legalidad y veracidad con el manejo presupuestal según administrativos financistas de la Universidad Nacional de Ucayali. 


\section{REFERENCIAS BIBLIOGRÁFICAS}

Albi, E., Contreras, C., González-Páramo, J. y Zubiri, I. (1992). Teoría de la hacienda pública. Barcelona: Ariel.

Arbildo, A. (2018). Administración financiera gubernamental y utilización de fondos públicos en la percepción de los trabajadores administrativos del Ministerio Público-Fiscalía de la Nación, Distrito Fiscal de Ucayali, 2018. Tesis de maestría. Universidad César Vallejo, Trujillo.

Bulacio, J. (2000). La ley de Wagner y el gasto público en Argentina. Recuperado el 23 de junio de 2018. Disponible en: www.aaep.org.ar/anales/works

Carranza, E. (2017). Eficiencia administrativa en la ejecución de las obras públicas y la gestión de contratos de la Gerencia de Infraestructura del Ministerio Público Fiscalía de la Nación. Tesis de maestría. Universidad Inca Garcilaso de la Vega, Lima. Disponible en: repositorio.uigv.edu.pe

Castro, C.; Villacorta, J. \& Caja, T. (2017). Remuneración y calidad de servicio en el hospital regional de Pucallpa, 2016. Tesis de licenciatura. Universidad Nacional de Ucayali, Pucallpa: Disponible en: http://repositorio.unu.edu.pe/

Cañas, A. (2015). Discrecionalidad y compensación del gasto público en gobiernos estatales. Tesis de doctorado. Centro de investigación y docencias económicas, México. Disponible en: repositorioinstitucional.mx

Constitución política del Perú 1993. http://www.congreso.gob.pe/Docs/files/ documentos/constitucionparte1993-12-09-2017.pdf

Criollo, G. (2018). Calidad del servicio asistencial y gestión comunitaria en la percepción de los usuarios del Seguro Integral de Salud, Hospital Amazónico-Yarinacocha, Ucayali, 2018. Tesis de maestría. Universidad César Vallejo, Trujillo.

Decreto legislativo del Sistema nacional de presupuesto público $N^{\circ} 1440$.

Dirección general de presupuesto público (2011). El sistema nacional de presupuesto. Guía básica. Lima: Ministerio de Economía y Finanzas.

González, P. \& Rodríguez, G. (2013). Análisis de la eficiencia del gasto público: Universidad de Cartagena. 2008-2011. Tesis de titulación. Colombia: Universidad de Cartagena. Disponible en: repositorio.unicartagena.edu.co

Letelier, F. (2016). Efectos del gasto público y de su composición en el crecimiento económico. Tesis de maestría. Chile: Pontificia Universidad Católica de Chile. Disponible en: http://economia.uc.cl/wp-content/uploads/2017/07/tesis-Letelier-2016.pdf

Ley № 28411. Ley General del Sistema de Presupuesto Público. http://transparencia.mtc.gob.pe/ idm_docs/normas_legales/1_0_31.pdf

Panduro, K. (2018). Gestión bajo el enfoque de liderazgo, en las mypes del sector construcción, ciudad de Pucallpa, año 2018. Tesis de doctorado. Universidad Católica los Ángeles de Chimbote. Pucallpa. Disponible en: http://repositorio.uladech.edu.pe/

Patiño, K. (2018). Gestión presupuestal y satisfacción laboral en la percepción del personal administrativo, Ministerio Público sede Ucayali, 2018. Tesis de maestría. Universidad César Vallejo, Trujillo. 
María M. Castillo

Ponce, S. (2007). Eficiencia del gasto público en educación: un análisis por departamentos. Tesis de licenciatura. Pontificia Universidad Católica del Perú, Lima. Disponible en: tesis.pucp.edu.pe

Rodríguez, O. (2017). Evaluación de la ejecución presupuestal de la Policía Nacional del Perú y su implicancia en la Política Pública de Seguridad Ciudadana. Tesis de maestría. Pontificia Universidad Católica del Perú, Lima. Disponible en: tesis.pucp.edu.pe 